\title{
Flaring of Ala Nasi: A Reliable Diagnostic Sign for Abductor Spasmodic Dysphonia
}

\author{
Jayakumar R Menon \\ Consultant Laryngologist, Head, Department of ENT, Kerala Institute of Medical Sciences, Thiruvananthapuram, Kerala, India
}

Correspondence: Jayakumar R Menon, Consultant Laryngologist and Head, Department of ENT, Kerala Institute of Medical Sciences, Thiruvananthapuram, Kerala, India, e-mail: jrmenon@ rediffmail.com

\section{ABSTRACT}

A prospective double blinded controlled study was done to assess the relation between flaring of ala nasi and abductor spasms in abductor spasmodic dysphonia. All the 24 cases of clinically and stroboscopically proven cases of abductor spasmodic dysphonia were found to have visible and easily detectable flaring of ala nasi during the abductor spasm. Among the control group of 24 dysphonia patients, only two cases showed this sign. Both were cases of vocal nodules. Rest of the vocal nodule cases in the study did not show this sign. Even the two cases which demonstrated this particular finding had the ala nasi flaring almost persistently during the phonation, as opposed to the constantly recurring pattern found in abductor spasmodic dysphonia. The study found definite association between the clinical sign of flaring of ala nasi and abductor spasmodic dysphonia.

Objective: To study the reliability of flaring of ala nasi as a diagnostic sign in abductor spasmodic dysphonia.

Keywords: Spasmodic dysphonia, Flaring, Ala nasi.

\section{INTRODUCTION}

A bductor spasmodic dysphonia constitutes roughly $15 \%$ of laryngeal dystonias. ${ }^{1}$ The gold standard of evaluation of spasmodic dysphonia is fiberoptic laryngoscopy. ${ }^{2}$ Stroboscopy gives more details regarding the changes in the wave pattern during each spasms. Kouffman suggested that the use of fiberoptic scope of the larynx as well as voice analysis. ${ }^{3}$

The abductor spasmodic dysphonia is characterized by spasms of posterior cricoarytenoid muscle (PCA) thus producing a breathy, effortful and hypophonic voice with abrupt termination of voicing causing characteristic aphonic or whispered segments of speech. The laryngoscopic examination shows a synchronous and untimely abduction of the true vocal folds that cause an extremely wide open glottic chink. ${ }^{4}$ These spasms are triggered by consonant sounds especially fricative ones. R ole of laryngeal EM G is inconclusive. The author has found a unique clinical sign in all the cases of abductor spasmodic dysphonia. Synchronous with the abductor spasms, there was flaring of ala nasi.

\section{MATERIALS AND METHODS}

This was a prospective double blinded controlled clinical trial conducted in the author's voice clinic during the period J anuary 2007 to December 2009. The study group included 24 cases of abductor spasmodic dysphonia that came for treatment during the study period. The diagnosis was made by perceptual analysis and video stroboscopic examination of the larynx. Only those patients who showed the abductor spasms were included in the study. The youngest patient was 24-year-old and the oldest 70-year-old. Seventeen of them were males and seven were females. The control group was selected from similar age and

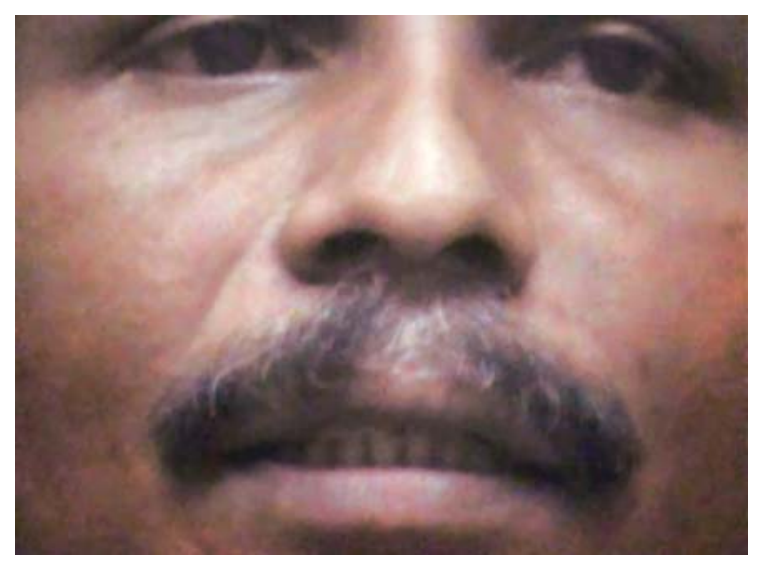

Fig. 1: Ala nasi without flaring

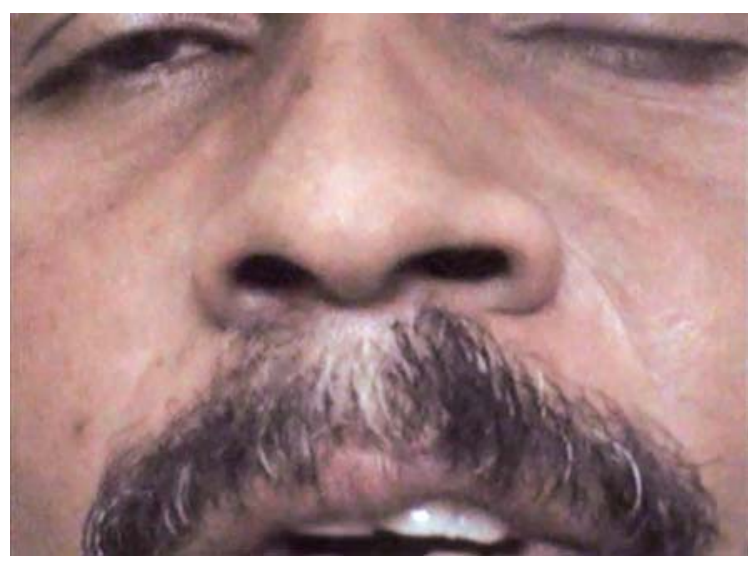

Fig. 2: Flared ala nasi during abductor spasm

sex group of patients who attended the clinic during the study period with complaints of voice problem. They were selected on a random basis. They all underwent stroboscopic examination 
of the larynx by the author and a diagnosis was reached. B oth the groups of patients were assessed by a clinician, who was not an ENT special ist, to whom the diagnosis was not reveal ed. A II the patients were examined for any recurring movement of the ala nasi during phonation. B oth flaring as well as constriction of the ala were noted and documented (Figs 1 and 2).

\section{RESULTS}

All the 24 patients in the study group unequivocally demonstrated the flaring of ala nasi repeatedly during phonation. There was no difficulty in identifying this clinical sign. Even a nonmedical person was able to identify it. How ever, there was no persistent flaring of the ala nasi throughout the duration of speech. Newly diagnosed as well as old cases of abductor spasmodic dysphonia all showed this sign infallibly. Even previous successful treatment with botulinum toxin injection to posterior cricoarytenoid muscle (PCA) did not have any bearing on this sign. A mong the control group, there were 11 cases of vocal nodules, three cases of puberphonia, two cases of sulcus vocal is, two cases of ventricular dysphonia, two cases of abductor spasmodic dysphonia and one case each of unilateral vocal cord palsy, vocal cord polyp, vocal cord cyst and cleft palate repaired. Of them, two cases of vocal nodules showed flaring of ala nasi. But as opposed to abductor spasmodic dysphonia, this was a persistent rather than paroxysmal finding. N one of the other cases showed any flaring of ala nasi during any stage of voice production. Interestingly, one case of sulcus vocalis and the case of repaired cleft palate both showed ala nasi constriction during initial phase of voicing. The two vocal nodule patients, who showed flaring of ala nasi, did not have this clinical sign when they had completed the voice therapy. The sulcus vocalis patient as well as the cleft palate patient also had significant reduction in the ala nasi contraction after surgical treatment and voice therapy.

\section{DISCUSSION}

Though supplied by different cranial nerves, both dilator ala nasi (nerve supply CN V II) and posterior cricoarytenoid (PCA) (nerve supply $\mathrm{CN} X$ through $\mathrm{RLN}$ ) are inspiratory muscles. Their nuclei are close to each other in the brain stem. The physiological inter-relation between nose and laryngeal function has been studied and proven in detail by Kratschmer. ${ }^{5}$ This relation can be illustrated by a simple clinical test. If one intentionally flares the ala nasi during phonation, it can be noted that the loudness of the voice comes down appreciably. It can be observed that the volume will go up only when the ala nasi are brought back to the original position. ${ }^{6}$ A ctually there are other physiological examples to this particular association. It is virtually impossible to complete the act of swallowing, if the alae nasi are kept in the flared position. Similarly in patients with respiratory distress, it is a common finding to see the ala nasi flaring.
Generally, the abductor PCA is suppressed during phonation. ${ }^{7}$ In the light of the above-mentioned physiology, the occurrence of the flaring of ala nasi during the abductor spasms in abductor spasmodic dysphonia is perfectly understandable. The two cases of vocal nodules that showed al a nasi flaring again probably underlines this relation. Though included in the hyperkinetic voice disorders, every vocal nodule patients on diagnostic laryngoscopy demonstrates an hour glassshaped phonatory gap. The width of the gap varies from patient to patient. A nd, it is a well-known fact that, vocal nodule patients with severe muscle tension dysphonia will have marked breathiness of voice. The two vocal nodule patients, who demonstrated the ala nasi flaring, had significant breathiness of voice and wider phonatory gap on laryngoscopy. It is interesting to note that, both these patients after successful voice therapy did not have either the ala nasi flaring or phonatory gap. Similarly, the opposite reaction of constriction of ala nasi in velopharyngeal incompetence is easily explained. The ala nasi constriction in one case of sulcus vocal is needs further studies for evaluation. It is quite possible that, here there is a structural defect in the vocal cords resulting in air leak as opposed to the unintentional abductor muscular activity happening in abductor spasmodic dysphonia and severe muscle tension dysphonia. A gain it was worthwhile to note that the ala nasi constriction almost completely disappeared after several voice therapy sessions. This led the author to use a voice therapy technique in which the abductor spasmodic dysphonia patients were instructed to control their flaring of ala nasi intentionally with the help of visual feedback. The initial response has been extremely encouraging.

The fact that all the cases in the study group demonstrated this particular sign is statistically significant. Even the two cases in the control group who showed this sign was different in the sense that the flaring was not only of a paroxysmal nature but also more of a continuous type. W e can safely assume that all cases of abductor spasmodic dysphonia will show this sign, though all cases showing flaring of al a nasi may not be abductor spasmodic dysphonia. But close scrutiny reveals that the ala nasi flaring was typically paroxysmal in abductor spasmodic dysphonia and is unique to the condition. The only problem remaining is the mixed spasmodic dysphonia. How those cases demonstrate this sign needs further studies.

\section{CONCLUSION}

All cases of abductor spasmodic dysphonia show the unmistakable clinical sign of paroxysmal flaring of ala nasi during the abductor spasms. This can be considered as a definite diagnostic sign of the disorder. Occasionally, flaring of ala nasi can occur in severe muscle tension dysphonia associated with vocal nodules. However, it is clearly distinguishable from the ala nasi flaring in abductor spasmodic dysphonia, being rather continuous than paradoxical in nature. $V$ isual biofeedback voice therapy is a possible treatment option of abductor spasmodic 
dysphonia. A bove all this study reiterates the fact that examining the voice and voice box alone is not sufficient in the diagnosis and management of voice disorders.

\section{REFERENCES}

1. Blitzer A, B rin M F. In L arynx A multidisciplinary approach by Marvin P Fried (2nd ed). Mosby Publishers 1995; 187-95.

2. Woodson G. U se of fibreoptic laryngoscopy to assess patients with spasmodic dysphonia. J V oice 1991;5:85.

3. Koufman J. A new classification of laryngeal dystonias. The visible voice 1992;1:1-5.
4. Blitzer A, Gibbs SR. Spasmodic dysphonia in the larynx by Ossoff Robert $\mathrm{H}$ et al (1st ed), Lippincot publishers 2003: 259-68.

5. Kratschmer $F$. On reflexes from the nasal mucous membrane on respiration and circulation. Respiratory Physiology 2001;127:93-104.

6. Menon JR. Flaring of ala nasi-a diagnostic sign for abductor spasmodic dysphonia. Programme and abstract book, 3rd world voice congress 2006:052.

7. Ortega JD, et al. B rain stem mechanism of laryngeal control as revealed by microstimulation studies. V ocal physiology: V oice production, mechanism and functions edited by Osamu Fujimura $1988 ; 2: 19-28$. 